\title{
Níveis de glicerina para novilhas suplementadas em pastagens: desempenho, ingestão, eficiência alimentar e digestibilidade
}

\section{Glycerine levels for crossbred heifers growing in pasture: performance, feed intake, feed eficiency and digestibility}

\author{
Mariana de Souza Farias ${ }^{1}$; Ivanor Nunes do Prado ${ }^{2 *}$; \\ Maribel Velandia Valero'; Fernando Zawadzki ${ }^{1}$; Robério Rodrigues Silva ${ }^{3}$; \\ Carlos Emanuel Eiras ${ }^{1}$; Dayane Cristina Rivaroli ${ }^{4}$; Beatriz Silva Lima ${ }^{4}$
}

\section{Resumo}

O objetivo deste trabalho foi avaliar a inclusão de glicerina sobre o desempenho animal, eficiência alimentar e digestibilidade em novilhas suplementadas em pastagem de Brachiaria brizantha cultivar Marandu. O período experimental foi de 102 dias, sendo os primeiros 14 destinados à adaptação às dietas e às condições experimentais. Foram utilizadas 36 novilhas mestiças com peso inicial médio de $226 \pm 12 \mathrm{~kg}$ e 13 meses de idade distribuídas em delineamento inteiramente casualizado com quatro tratamentos e nove repetições: G00 = controle; G2,8 $=2,8 \%$; G6,1 $=6,1 \%$ e G9,0 $=9,0 \%$ de glicerina na matéria seca (MS) total na dieta. As novilhas foram pesadas a cada 28 dias para avaliação do ganho médio diário. A ingestão de MS foi estimada a partir da produção fecal, com uso de óxido crômico $\left(\mathrm{Cr}_{2} \mathrm{O}_{3}\right)$ como indicador externo e da MS indigestível (MSi) como indicador interno. A digestibilidade aparente foi estimada a partir da produção fecal determinada pelo óxido crômico. A adição de glicerina na dieta determinou redução linear $(\mathrm{P}<0,05)$ no peso final e ganho médio diário das novilhas. A ingestão diária de MS do suplemento, da pastagem e total foi semelhante $(\mathrm{P}>0,05)$ entre os tratamentos. A ingestão de extrato etéreo aumentou de forma linear $(\mathrm{P}<0,05)$ conforme houve acréscimo de glicerol na dieta. A ingestão de carboidratos apresentou redução linear $(\mathrm{P}<0,05)$ em função dos níveis de glicerol às dietas. A inclusão de glicerina não teve efeito $(\mathrm{P}>0,05)$ sobre os coeficientes de digestibilidade da $\mathrm{MS}, \mathrm{PB}, \mathrm{MO}, \mathrm{FDN}, \mathrm{CNF}$ e CT. A inclusão de glicerina às dietas aumentou de forma linear $(\mathrm{P}<0,05)$ a digestibilidade do extrato etéreo. O uso de glicerina de baixa pureza não é recomendado, pois determina redução no desempenho animal.

Palavras chave: Desempenho animal, bovinos, eficiência alimentar, glicerina

\begin{abstract}
This work was carried out to study the effect of levels different addition of glycerine on animal performance, feed efficiency and digestibility of heifers supplemented in Brachiaria brizantha cultivar Marandu pasture. The experimental period was 102 days. The first 14 days was the period of adaptation of heifers to experimental diets and management. It was used 36 crossbred heifers with an average initial
\end{abstract}

\footnotetext{
${ }^{1}$ Mestre em Zootecnia, Programa de Pós Graduação em Zootecnia, Universidade Estadual de Maringá, UEM, Maringá, PR. Av. Colombo, 5790,CEP 87.020-900.E-mail: marianafarias@gmail.com

2 Prof. Titular, Dept ${ }^{\circ}$ de Zootecnia, UEM, Programa de Pós Graduação em Zootecnia. Maringá, PR. Pesquisador IA do CNPq. E-mail: inprado@uem.br

${ }^{3}$ Prof. do Dept ${ }^{\mathrm{o}}$ de Zootecnia, Universidade Estadual do Sudoeste da Bahia, UESB, Programa de Pós Graduação em Zootecnia, Salvador, BA. E-mail: rrsilva.uesb@hotmail.com

${ }^{4}$ Discente de Graduação, Curso de Zootecnia, UEM, Maringá, PR. E-mail: dayrivaroli@hotmail.com

*Autor para correspondência
} 
weight of $226 \pm 12 \mathrm{~kg}$ and 13 months old. The heifers were distributed in a completely randomized design with four treatments and nine replicates: control $=\mathrm{G} 00, \mathrm{G} 2.8=2.8 \%, \mathrm{G} 6.1=6.1 \%$ and $\mathrm{G} 9.9=9.9 \%$ of glycerine in dry matter of diet. The heifers were weighed every 28 days for evaluation of average daily gain (ADG). The dry matter intake (DMI) was estimated from fecal output, recorded with the aid of chromic oxide $\left(\mathrm{Cr}_{2} \mathrm{O}_{3}\right)$ as an external and indigestible dry matter (DMi) as marker internal. The apparent digestibility was estimated from fecal output determined by chromic oxide. The addition of glycerine in the diet caused a linear reduction $(\mathrm{P}<0.05)$ in final weight $(\mathrm{FLW})$ and average daily gain (ADG) of heifers. The daily intake of dry matter $(\mathrm{kg} / \mathrm{day})$ of supplementation, pasture and total was similar $(\mathrm{P}>$ $0.05)$ among treatments. The intake of ether extract increased linearly $(\mathrm{P}<0.05)$ as there was an increase of glycerol in the diet. Carbohydrate intake decreased linearly $(\mathrm{P}<0.05)$ depending on the levels of glycerine to diets. The inclusion of glycerine had no effect $(\mathrm{P}>0.05)$ on the digestibility of dry matter, organic matter, neutral detergent fiber, crude protein, non-fibrous carbohydrates and carbohydrates total. Moreover, the inclusion of glycerine in the diets increased linearly $(\mathrm{P}<0.05)$ the digestibility of ether extract. The inclusion of low-purity glycerine is not recommended because it provides a reduction in animal performance.

Key words: Animal performance, cattle, feed efficiency, glycerol

\section{Introdução}

O consumo de forragem de animais em pastejo é influenciado por três grupos de fatores: os que afetam o processo de digestão, os que afetam o processo de ingestão e aqueles que afetam as exigências nutricionais e a demanda por nutrientes (BERCHIELLI; PIRES; OLIVEIRA, 2006). Moreira et al. (2003) relataram que um dos problemas existentes na criação de bovinos em pastagens é a variação tanto na quantidade como na qualidade da matéria seca produzida com efeito negativo na produtividade animal. Neste contexto, a suplementação dos animais em pastejo tem se constituído numa das principais alternativas economicamente viáveis de produção de bovinos de corte em pastagens naturais ou cultivadas. Nas épocas do ano em que a produção de forragem é menor em termos quantitativos e qualitativos, a suplementação dos animais em pastejo possibilita a obtenção de maiores ganhos de peso por animal e por área (MOREIRA et al., 2004). Desta forma, um dos objetivos básicos de todo sistema de produção de bovinos em pastagem, é suprir as exigências nutricionais dos animais ao longo do ano, mantendo uma oferta permanente de alimento em quantidade e qualidade suficientes, com a finalidade de obter resposta produtiva satisfatória.

Por outro lado, o sistema de terminação de bovinos em confinamento pode proporcionar ganho de peso adequado e carne de melhor qualidade (MAGGIONI et al., 2009; ROTTA et al., 2009a, b; DUCATTI et al., 2009; Prado et al., 2009), porém, apresenta maior custo de produção (ABRAHÃO et al., 2005; ARICETTI et al., 2008). Da mesma forma, a terminação de novilhas com uso de milho como fonte de energia proporciona bom ganho em peso, mas com aumento no custo de produção (D'OLIVEIRA et al., 1997; KAZAMA et al., 2008). Assim sendo, fontes alternativas de energia são pesquisadas para substituir o milho na recria e terminação de bovinos uma vez que este alimento é valorizado na alimentação de monogástricos e humanos.

A glicerina é um coproduto da indústria do biodiesel (ABDALLA et al., 2008). Para cada 90 $\mathrm{m}^{3}$ de biodiesel produzidos são gerados $10 \mathrm{~m}^{3}$ de glicerina (DASARI et al., 2005). A exigência, por parte do Programa Nacional de Produção e Uso de Biodiesel do Governo Federal, de adição do biodiesel ao óleo diesel será de 5\% a partir de 2013. Desta forma, serão necessários cerca de 2,5 bilhões de litros de biodiesel para cumprir a Lei 11097/2005 (ABDALLA et al., 2008). As indústrias de tabaco, alimentos, bebidas e cosméticos (DASARI et al., 2005) não conseguirão aproveitar todo excedente, sendo necessário encontrar novas aplicações para a glicerina. 
Os ruminantes têm capacidade de utilizar o glicerol presente na glicerina como precursor gliconeogênico (CHUNG et al., 2007) para a manutenção dos níveis plasmáticos de glicose. $\mathrm{O}$ glicerol é convertido em glicose, pois este entra na forma de fosfato di-hidroxicetona e é convertido em 3-fosfoglicerato pela ação enzima glicerol-3-fosfato desidrogenase para entrar na via gliconeogênica (KREHBIEL, 2008). O fornecimento de glicerina tende a reduzir a quantidade disponível de carbono e hidrogênio para produção de gás metano, devido ao aumento de propionato (TRABUE et al., 2007).

A glicerina possui natureza higroscópica, o que pode aumentar a capacidade de retenção de água das rações em ambientes de baixa umidade e melhorar a palatabilidade do concentrado devido ao seu aroma suave e sabor adocicado, além de aumentar o consumo de concentrado (ELAM et al., 2008). A glicerina apresenta composição química muito variável, uma vez que varia com a matéria-prima utilizada para produção de biodiesel (VIEIRA et al., 2005; ELAM et al., 2008).

A inclusão de 7,5 e 15,0\% de glicerina bruta na matéria seca para novilhas em confinamento reduziu o desempenho animal segundo Elam et al. (2008). Esse menor ganho de peso pode ser explicado pela redução na ingestão de matéria seca e pela mudança no comportamento alimentar das novilhas. Parsons, Shelor e Drouillard (2009) avaliaram teores de 0, 2, 4, 8, 12 e 16\% de inclusão de glicerina na dieta de novilhas mestiças e observaram ganhos de peso de 1,$19 ; 1,34 ; 1,29 ; 1,25 ; 1,17$ e $1,03 \mathrm{~kg} / \mathrm{dia}$, respectivamente. Estes autores concluíram que até $8 \%$ de inclusão de glicerina com base na matéria seca melhora o desempenho animal. Da mesma forma, Mach, Bach e Devant (2009) avaliaram quatro porcentagens de inclusão de glicerina ( 0 , 4, 8 e $12 \%$ de glicerina com 3,47 Mcal/kg na MS) sobre o desempenho de machos não castrados holandeses alimentados com dieta de alto concentrado e encontraram ganho de peso de 1,29 e 1,43 kg/dia para 12 e $4 \%$ de inclusão, respectivamente.
O objetivo deste trabalho foi avaliar o efeito da inclusão de glicerina na suplementação de novilhas mestiças criadas em pastagem de Brachiaria brizantha cv. Marandu e suas implicações sobre o consumo, digestibilidade dos nutrientes e no desempenho.

\section{Material e Métodos}

O experimento foi desenvolvido na Fazenda Princesa do Mateiro situada no município de Ribeirão do Largo no Estado da Bahia no período de setembro a dezembro de 2009. O período experimental foi de 102 dias, sendo os primeiros 14 dias para adaptação das novilhas às dietas e às condições experimentais. A área experimental foi dividida em 10 piquetes de aproximadamente 1,8 ha cada, totalizando 18 ha de pastagem formada por Brachiaria brizantha cultivar Marandu. Foram utilizadas 36 novilhas mestiças com peso inicial médio de $226 \pm 12 \mathrm{~kg}$ e 13 meses de idade distribuídas em delineamento inteiramente casualizado com quatro tratamentos e nove repetições: $\mathrm{G} 00=$ controle; $\mathrm{G} 2,8=2,8 \%$; G6, 1 $=6,1 \%$ e $\mathrm{G} 9,0=9,0 \%$ de glicerina na matéria seca total da dieta. O suplemento foi fornecido em cocho de plástico sem cobertura uma vez ao dia (10:00 h). A quantidade diária de suplemento oferecida aos animais dos tratamentos foi o somatório das quantidades que deveriam ser fornecidas para cada animal, pois os animais foram alimentados em grupo. Os animais foram alternados nos piquetes a cada 7 dias. Na Tabela 1 está apresentada a composição percentual dos suplementos com base na matéria seca. A glicerina utilizada apresentava $16 \%$ de metanol e para a eliminação deste composto a glicerina foi aquecida a $75^{\circ} \mathrm{C}$ antes de ser misturada às dietas. $\mathrm{O}$ aquecimento da glicerina foi realizado em fogão a gás por um período de 30 minutos. Foi utilizado glicerol de pureza media proveniente da extração de óleo vegetal e de gordura animal com $12,5 \%$ de água, $81,2 \%$ de glicerol e $0,33 \%$ de metanol. 
Tabela 1. Composição percentual dos concentrados.

\begin{tabular}{|c|c|c|c|c|}
\hline \multirow{2}{*}{ Ingredientes \% } & \multicolumn{4}{|c|}{ Níveis de glicerina (\% da MS) } \\
\hline & G00 & $\mathrm{G} 2,8$ & G6,1 & $\mathrm{G} 9,0$ \\
\hline Milho moído & 80,50 & 74,84 & 68,38 & 61,52 \\
\hline Farelo de soja & 16,00 & 17,67 & 20,09 & 22,78 \\
\hline Ureia & 2,00 & 2,09 & 2,17 & 2,29 \\
\hline Sal mineral ${ }^{1}$ & 1,50 & 1,55 & 1,62 & 1,71 \\
\hline Fosfato bicálcico & 0,00 & 0,52 & 1,08 & 1,13 \\
\hline Calcário & 0,00 & 0,00 & 0,00 & 0,58 \\
\hline Glicerina & 0,00 & 2,80 & 6,10 & 9,00 \\
\hline
\end{tabular}

${ }^{1}$ Níveis de garantia (por kg): cálcio - 175 g; fósforo - 100 g; sódio - 114 g; selênio - 15 g; magnésio - 15 g; zinco - 6.004 mg; manganês - $1.250 \mathrm{mg}$; cobre - 1.875; iodo - $180 \mathrm{mg}$; cobalto - $125 \mathrm{mg}$; selênio - $30 \mathrm{mg}$; flúor (máximo) - $1.000 \mathrm{mg}$.

Fonte: Elaboração dos autores.

As novilhas foram pesadas ao início e ao final do experimento. Também foram realizadas pesagens intermediárias a cada 28 dias para ajuste da quantidade de ração ofertada, avaliação do ganho médio diário de peso e estimativa da conversão e eficiência alimentar. $\mathrm{O}$ ganho médio diário (GMD) foi determinado pela diferença entre o peso vivo inicial (PVI) e o peso vivo final (PVF) dividido pelo período experimental em dias.

A conversão alimentar da MS (CAMS) foi calculado em função do consumo e do desempenho animal conforme a equação: CAMS = (IDMS/ GMD). IDMS = ingestão diária de matéria seca $(\mathrm{kg}$ $\mathrm{MS} / \mathrm{dia})$ e GMD = ganho médio diário $(\mathrm{kg} / \mathrm{dia})$.

Tabela 2. Composição química da Brachiaria brizanta cv. Marandu e dos concentrados (\% de MS), disponibilidade total de matéria seca, biomassa residual, taxa de lotação, taxa de acúmulo e oferta de forragem.

\begin{tabular}{lrrrrr}
\hline \multirow{2}{*}{ Ingredientes } & Brachiaria & \multicolumn{4}{c}{ Níveis de glicerina (\% da MS) } \\
\cline { 2 - 6 } & brizantha & G0,0 & G2,8 & \multicolumn{1}{c}{ G6,1 } & G9,0 \\
\hline Matéria seca, \% & 90,16 & 91,66 & 92,37 & 91,83 & 92,23 \\
Proteína bruta, \% & 5,62 & 21,07 & 22,13 & 23,27 & 24,65 \\
Extrato etéreo, \% & 1,20 & 2,47 & 2,72 & 2,79 & 2,82 \\
Carboidratos totais, \% & 84,30 & 72,80 & 71,00 & 68,92 & 66,70 \\
Carboidratos não fibrosos, \% & 19,13 & 58,90 & 57,33 & 55,50 & 53,42 \\
Energia bruta, kcal/kg & 4224,56 & 4305,15 & 4242,60 & 4234,65 & 4197,13 \\
Fibra detergente neutro, \% & 66,52 & 13,90 & 13,67 & 13,42 & 13,28 \\
Fibra detergente ácido, \% & 40,51 & 3,59 & 3,79 & 3,92 & 4,17 \\
Nutrientes digestíveis totais, \% & 51,02 & 63,68 & 60,58 & 61,38 & 61,38 \\
Cinzas, \% & 8,87 & 3,66 & 4,15 & 4,99 & 5,82 \\
Disponibilidade total de MS, kg/ha & 3103,54 & - & - & - & - \\
Biomassa residual, kg de MS/ha/dia & 110,84 & - & - & - & - \\
Taxa de lotação, UA/há & 1,33 & - & - & - & - \\
Taxa de acúmulo, kg MS/ha/dia & 27,71 & - & - & - & - \\
Oferta de forragem, kg MS/100 kg PV/dia & 23,19 & - & - & - & - \\
\hline
\end{tabular}

Fonte: Laboratório de Alimentos e Nutrição da Universidade Estadual de Maringá. 
A eficiência alimentar foi calculada em função do desempenho animal e o consumo conforme a equação: $\mathrm{EAL}=(\mathrm{GMD} / \mathrm{IDMS}) . \mathrm{GMD}=$ ganho médio diário $(\mathrm{kg} / \mathrm{dia})$ e IDMS = ingestão diária de matéria seca ( $\mathrm{kg} \mathrm{MS/} \mathrm{dia).}$

A pastagem foi avaliada a cada 28 dias. A taxa de lotação (TL) foi calculada considerando a unidade animal (UA) como sendo $450 \mathrm{~kg}$ de PV, utilizandose a seguinte fórmula: $\mathrm{TL}=(\mathrm{UAt}) /$ área em que $\mathrm{TL}$ = taxa de lotação (UA/ha); UAt = unidade animal total (kg); Área = área experimental total (ha). Para estimar a disponibilidade de MS, foram obtidas, com auxílio de um quadrado de $0,25 \mathrm{~m}^{2}$, lançado 12 vezes, ao acaso, em cada piquete amostras de forragem, cortadas rente ao solo, conforme metodologia descrita por Wilm, Costelo e Klipple (1994). Foi adotado o método de lotação variável com mesma carga animal. Para reduzir a influência da variação de biomassa entre piquetes, as novilhas permaneceram em cada piquete por sete dias e, após esse período, foram transferidas para outro, em sentido aleatório. As estimativas de biomassa residual diária (BRD) foram realizadas, conforme o método da dupla amostragem proposto por Wilm, Costelo e Klipple (1944). Antes do corte, foi estimada visualmente a matéria seca da biomassa da amostra. Foram utilizados os valores das amostras cortadas e estimadas visualmente quando foi jogado 40 vezes o quadrado e, posteriormente, foi calculada a biomassa de forragem expressa em $\mathrm{kg} / \mathrm{ha}$ pela equação proposta por Gardner (1986).

O acúmulo de MS, nos diferentes períodos experimentais, foi calculado multiplicando-se o valor da taxa de acúmulo diário (TAD) de MS pelo número de dias do período. A estimativa da TAD foi realizada pela equação proposta por Campbell (1966): $\operatorname{TAD}_{\mathrm{J}}=\left(\mathrm{G}_{\mathrm{i}}-\mathrm{F}_{\mathrm{i}}-1\right) / \mathrm{n}$, em que $\mathrm{TAD}_{\mathrm{j}}=$ taxa de acúmulo de matéria seca diária $(\mathrm{kg} \mathrm{MS} / \mathrm{ha} / \mathrm{dia})$ no período $\mathrm{j}, \mathrm{G}_{\mathrm{i}}=\mathrm{MS}$ final $(\mathrm{kgMS} / \mathrm{ha})$ média dos quatro piquetes vazios no instante $i, F_{i}-1=M S$ inicial ( $\mathrm{kg} \mathrm{MS} / \mathrm{ha}$ ) média presente nos piquetes vazios no instante $\mathrm{i}-1, \mathrm{n}=$ número de dias do período j. A oferta de forragem (OF) foi calculada de acordo com a fórmula: $\mathrm{OF}=\{(\mathrm{BRD} *$ área $+\mathrm{TAD} *$ área $) / \mathrm{PV}$ total $\} * 100 . \mathrm{OF}=$ oferta de forragem $(\mathrm{kg} \mathrm{MS} / 100 \mathrm{~kg}$ $\mathrm{PV} /$ dia); $\mathrm{BRD}=$ biomassa residual total $(\mathrm{kg} \mathrm{MS} / \mathrm{ha} /$ dia), TAD = taxa de acúmulo diário ( $\mathrm{kg} \mathrm{MS/ha/dia),}$ $\mathrm{PV}=$ peso vivo dos animais ( $\mathrm{kg} / \mathrm{ha})$. As amostras de forragem coletadas pela dupla amostragem foram pesadas individualmente no campo e desse material foram retiradas sub amostras nas quais foram realizadas separação dos componentes estruturais: lâmina foliar (LF), colmo (C) e material morto (MM). As amostras de forragem foram pré-secas em estufa de circulação forçada a ar a $55-65^{\circ} \mathrm{C}$ por $72 \mathrm{~h}$ para determinação do peso seco.

A ingestão de matéria seca (IMS) foi estimada a partir da produção fecal, verificada com auxílio de óxido crômico $\left(\mathrm{Cr}_{2} \mathrm{O}_{3}\right)$ como indicador externo e da matéria seca indigestível (MSi) como indicador interno, 60 dias após o início do experimento. Foi fornecida uma dose diária de $10 \mathrm{~g}$ de óxido crômico direto na boca do animal (09:00 h) durante doze dias, sendo que, os sete primeiros dias constituíram o período de adaptação dos animais ao manejo e a regulação da excreção de cromo nas fezes. A ingestão individual de suplemento foi estimada pela utilização do indicador externo dióxido de titânio $\left(\mathrm{TiO}_{2}\right)$ administrado em dose diária na proporção de $10 \mathrm{~g}$ por animal misturado ao concentrado. Considerando nove animais por tratamento foram misturados $90 \mathrm{~g}$ de $\mathrm{TiO}_{2}$ por dia, durante o mesmo período de fornecimento do óxido crômico, com sete dias de adaptação e cinco dias de coleta de fezes. As fezes foram coletadas (aproximadamente 300g) na pastagem logo após a excreção, identificadas e congeladas.

As amostras foram secas em estufa de ventilação forçada e trituradas para obtenção de amostras compostas. As amostras de fezes foram analisadas quanto aos teores de cromo conforme metodologia descrita por Kimura e Miller (1967) utilizando-se digestão nitroperclórica e espectrofotometria de absorção atômica (EAA). As análises de dióxido de titânio foram realizadas segundo Myers et al. (2004). A determinação da produção fecal foi realizada 
conforme a equação abaixo: $\mathrm{PF}=\mathrm{OF} / \mathrm{COF}$ em que $\mathrm{PF}$ é a produção fecal diária (g/dia); OF óxido crômico fornecido ( $\mathrm{g} / \mathrm{dia}$ ) e COF é a concentração de óxido crômico nas fezes (g/g MS). A ingestão individual de concentrado foi estimado dividindose a excreção total de $\mathrm{TiO}_{2}$ pela sua respectiva concentração no concentrado.

A digestibilidade aparente parcial e total foi estimada a partir da produção fecal determinada pelo óxido crômico. Para avaliação dos teores dos componentes indigestíveis, as amostras de forragem, fezes e de concentrado foram incubadas por 240 horas no rúmen de bovino fistulado da raça Purunã alimentado com silagem de milho e suplemento contendo $15 \%$ de glicerol em substituição ao milho com base na MS, na mesma proporção 60:40. As análises laboratoriais foram realizadas no Laboratório de Alimentos, Alimentação e Nutrição Animal do Departamento de Zootecnia da Universidade Estadual de Maringá.

As amostras de concentrado, forragem, fezes e os resíduos após incubação foram analisados para determinação dos teores de matéria seca (MS), proteína bruta (PB) e extrato etéreo (EE), conforme metodologia descrita por Silva e Queiroz (2002), fibra em detergente neutro (FDN) e fibra em detergente ácido (FDA) segundo Van Soest, Robertson e Lewis (1991). Os carboidratos totais
(CHOT) foram obtidos por diferença conforme a equação de Sniffen et al. (1992): CHOT = $100-$ $(\% \mathrm{~PB}+\% \mathrm{EE}+\% \mathrm{CZ})$. Os carboidratos não fibrosos $(\mathrm{CNF})$, pela diferença entre CHOT e FDN. Os teores de nutrientes digestíveis totais (NDT) e energia metabolizável foram obtidos conforme metodologia de Sniffen et al. (1992), sendo: NDT= (PBD + FDND + CNFD) + 2,25 (EED) em que PBD = ingestão de PB digestível; FDND = ingestão de FDN digestível; CNFD = ingestão de CNF digestível e $\mathrm{EED}=$ consumo de EE digestível. Para a energia metabolizável, considerou-se que $1,0 \mathrm{~kg}$ de NDT equivale a 4,409 Mcal de energia digestível e para a transformação em energia metabolizável utilizou-se o valor de $82 \%$ de eficiência de utilização de energia digestível. Os resultados das análises químicas da forragem, dos concentrados, a BRD, a TL, a TAD e a OF encontram-se na Tabela 2.

Os resultados foram interpretados estatisticamente por meio de equações de regressão, utilizando-se o SAS (2002).

\section{Resultados e Discussão}

O peso inicial $(226,4 \mathrm{~kg})$ foi semelhante entre os tratamentos (Tabela 3). O peso inicial foi obtido logo após o desmame até o momento da cobertura (300 kg).

Tabela 3. Peso inicial, final e ganho médio diário de novilhas alimentadas com diferentes teores de glicerina com suas respectivas equações de regressão e coeficientes de determinação $\left(r^{2}\right)$.

\begin{tabular}{lccccccc}
\hline & \multicolumn{4}{c}{ Níveis de glicerina, \% do MS ingerida } & \multirow{2}{*}{ Equação de Regressão } & \multirow{2}{*}{$\mathrm{r}^{2}$} \\
\cline { 2 - 5 } Variáveis & $\mathrm{G} 00$ & $\mathrm{G} 2,8$ & $\mathrm{G} 6,1$ & $\mathrm{G} 9,0$ & & \\
\hline Peso inicial, $\mathrm{kg}$ & 226,4 & 226,4 & 226,4 & 226,6 & & $\hat{\mathrm{Y}}=226,4$ & - \\
Peso final, $\mathrm{kg}$ & 305,8 & 302,0 & 297,8 & 289,3 & & $\hat{\mathrm{Y}}=305,8-5,50 \mathrm{x}$ & 0,96 \\
Ganho diário, $\mathrm{kg}$ & 0,78 & 0,74 & 0,70 & 0,62 & & $\hat{\mathrm{Y}}=0,78-0,05 \mathrm{x}$ & 0,92 \\
\hline
\end{tabular}

Fonte: Elaboração dos autores. 
A adição de glicerina na dieta determinou redução linear $(\mathrm{P}<0,05)$ no peso final $(\mathrm{PVF})$ e ganho médio diário (GMD) das novilhas suplementadas em pastagem de Brachiaria brizantha cv. Marandu (Tabela 3). Este comportamento foi semelhante ao encontrado por Elam et al. (2008) com GMD 1,64; 1,58 e $1,55 \mathrm{~kg} /$ dia para $0 ; 7,5$ e $15 \%$ de glicerina, respectivamente. Parsons, Shelor e Drouillard (2009) observaram resposta quadrática para o GMD de 1,$19 ; 1,34,1,29 ; 1,25 ; 1,17$ e 1,03 kg para $0 ; 2 ; 4$; $8 ; 12$ e $16 \%$ de glicerina na dieta, respectivamente. Por outro lado, Mach, Bach e Devant (2009) observaram que o GMD aumentou de acordo com a elevação dos teores de glicerina em relação à dieta controle até $10 \%$ de inclusão. Da mesma forma, Pyatt, Doane e Cecava (2007) observaram que o GMD foi $11,4 \%$ maior em bovinos alimentados com dietas de alto grão com inclusão de glicerina. No entanto, o ganho médio observado $(0,7 \mathrm{~kg} / \mathrm{dia})$ pode ser considerado adequado para novilhas de sobre ano (D'OLIVEIRA et al., 1997).

A redução no desempenho animal observado neste trabalho pode ser explicada pela qualidade da glicerina utilizada. A qualidade da glicerina pode variar em função do valor nutritivo da matéria prima e do processo de produção do biodiesel (VIEIRA et al., 2005). De modo geral, a glicerina é caracterizada como um produto de alta densidade, incolor, claro e de sabor adocicado (ELAM et al., 2008). No entanto, a glicerina usada neste experimento apresentava-se escura, pouco adocicada e adstringente ao paladar; características que podem ser atribuídas à presença de sais, impurezas e/ou reagentes usados no processo de transesterificação. Estas características da glicerina provavelmente determinaram redução do consumo de energia oriunda do suplemento das dietas com glicerina (Tabela 4) influenciando de maneira negativa o desempenho dos animais.

As ingestões diárias de MS, MO e PB não foram influenciadas $(\mathrm{P}>0,05)$ pelos teores de glicerina nas dietas das novilhas (Tabela 4). Elam et al. (2008), em experimento com bovinos alimentados com dietas contendo $0 ; 7,5$ e $15,0 \%$ de glicerina não observaram diferença na ingestão de MS. Da mesma forma, Mach, Bach e Devant (2009) observaram ingestão de MS de 8,18;8,19;8,53 e 8,19 kg/dia para os bovinos confinados e alimentados com teores de $0 ; 4 ; 8$ e 12\% de glicerina, respectivamente. Entretanto, alguns trabalhos indicam redução linear na ingestão de MS em função da adição de glicerina à dieta de bovinos como o experimento realizado por Parsons, Shelor e Drouillard (2009) em que os autores relataram ingestões de MS de 8,84; 8,88; 8,$66 ; 8,61 ; 8,40$ e $7,80 \mathrm{~kg} /$ dia para os teores de 0 , 2, 4, 8, 12 e 16\% de glicerina, respectivamente. Da mesma forma, Pyatt, Doane e Cecava (2007) observaram redução de 10\% quando utilizaram 10\% de inclusão de glicerina à dieta de bovinos. Segundo Parsons, Shelor e Drouillard (2009), a inclusão de até $5 \%$ de glicerina na dieta pode ser benéfica para o desempenho de bovinos, no entanto, concentrações superiores poderiam perturbar a microbiota ruminal.

A ingestão de EE aumentou de forma linear $(\mathrm{P}<0,05)$ com o aumento nos teores de glicerina na dieta (Tabela 4). Este aumento linear na ingestão de EE é explicado pela elevação do teor deste componente nutritivo na dieta de acordo com a inclusão de glicerina (Tabela 2). No entanto, o aumento de extrato etéreo na dieta não prejudicou a ingestão de alimentos, uma vez que teor do mesmo foi menor que $7 \%$ na matéria seca total, portanto, abaixo do nível que pode reduzir a ingestão de alimentos.

A ingestão de carboidratos não fibrosos e de carboidratos totais apresentou redução linear $(\mathrm{P}<0,05)$ em função dos teores de glicerina nas dietas (Tabela 4). Esta redução é explicada pela redução no teor de carboidratos das dietas com adição de glicerina (Tabela 2). Na realidade, a glicerina substituiu parte do milho nas dietas; portanto, a redução dos níveis de milho foi compensada pelo aumento dos níveis de glicerina. A glicerina é desprovida de carboidratos. Da mesma forma, Schröder e Südekun (1999) observaram redução de $0,7 \mathrm{~kg} /$ dia na ingestão de amido quando foram utilizados $15 \%$ de glicerina na dieta de bovinos em confinamento. 
Tabela 4. Ingestão dos componentes nutricionais e suas respectivas equações de regressão e coeficientes de determinação $\left(\mathrm{r}^{2}\right)$.

\begin{tabular}{|c|c|c|c|c|c|c|c|}
\hline \multirow{2}{*}{ Parâmetros } & \multicolumn{4}{|c|}{ Níveis de glicerina, $\%$ da MS ingerida } & \multirow{2}{*}{ Equação de Regressão } & \multirow{2}{*}{$\mathrm{CV}(\%)$} & \multirow{2}{*}{$r^{2}$} \\
\hline & G00 & $\mathrm{G} 2,8$ & G6,1 & $\mathrm{G} 9,0$ & & & \\
\hline IMSS, $\mathrm{kg} / \mathrm{dia}$ & 2,57 & 2,57 & 2,57 & 2,57 & $\hat{Y}=2,57$ & 22,30 & - \\
\hline IMSP, kg/dia & 3,90 & 3,48 & 3,32 & 3,42 & $\hat{\mathrm{Y}}=3,53$ & 24,68 & - \\
\hline IMST, $\mathrm{kg} / \mathrm{dia}$ & 6,47 & 6,43 & 5,89 & 5,99 & $\hat{\mathrm{Y}}=6,20$ & 17,34 & - \\
\hline IMST, \% do PV & 2,43 & 2,44 & 2,25 & 2,14 & $\hat{\mathrm{Y}}=2,31$ & 14,12 & - \\
\hline $\mathrm{IMO}, \mathrm{kg} / \mathrm{dia}$ & 6,01 & 5,43 & 5,12 & 5,05 & $\hat{\mathrm{Y}}=5,40$ & 17,34 & - \\
\hline $\mathrm{IPB}, \mathrm{kg} / \mathrm{dia}$ & 0,91 & 0,86 & 0,82 & 0,82 & $\hat{\mathrm{Y}}=0,86$ & 15,53 & - \\
\hline IEE, $\mathrm{kg} / \mathrm{dia}$ & 0,14 & 0,16 & 0,18 & 0,19 & $\hat{\mathrm{Y}}=0,143+5,069 x$ & 14,72 & 1,00 \\
\hline IFDN, $\mathrm{kg} / \mathrm{dia}$ & 2,48 & 2,22 & 2,11 & 2,15 & $\hat{Y}=2,24$ & 21,84 & - \\
\hline ICNF, $\mathrm{kg} / \mathrm{dia}$ & 2,48 & 2,19 & 2,08 & 1,96 & $\hat{\mathrm{Y}}=2,430-0,051 \mathrm{x}$ & - & 0,94 \\
\hline ICHOT, kg/dia & 4,96 & 4,42 & 4,20 & 4,11 & $\hat{\mathrm{Y}}=4,837-0,084 \mathrm{x}$ & - & 0,88 \\
\hline INDT, $\mathrm{kg} / \mathrm{dia}$ & 4,40 & 4,17 & 4,00 & 3,65 & $\hat{\mathrm{Y}}=4,418-0,073 \mathrm{x}$ & 15,08 & 0,98 \\
\hline IED, Mcal $/ \mathrm{kg}$ & 19,45 & 18,42 & 17,66 & 16,13 & $\hat{Y}=19,524-0,324 x$ & 15,08 & 0,98 \\
\hline IEM, Mcal $/ \mathrm{kg}$ & 15,95 & 15,11 & 14,48 & 13,23 & $\hat{Y}=16,001-0,265 x$ & 15,08 & 0,98 \\
\hline CAMS (kg/kg) & 8,29 & 8,59 & 8,41 & 9,66 & $\hat{\mathrm{Y}}=8,76$ & - & - \\
\hline
\end{tabular}

Ingestão de matéria seca do suplemento (IMSS), da pastagem (IMSP), total (IMST), IMST (\% do PV), matéria orgânica (IMO), proteína bruta (IPB), extrato etéreo (IEE), fibra em detergente neutro total (IFDN), carboidratos não fibrosos (ICNF), carboidratos totais (ICHOT), nutrientes digestíveis totais (INDT), energia digestível (IED), energia metabolizável (IEM) e conversão alimentar da matéria seca (CAMS).

Fonte: Elaboração dos autores.

Houve redução linear $(\mathrm{P}<0,05)$ na ingestão de NDT, energia digestível e energia metabolizável (Tabela 4). Estes resultados são explicados pela redução da quantidade de energia bruta na dieta (Tabela 2) em função da substituição parcial do milho pela glicerina. A glicerina é produzida como resultado da transesterificação de triglicerídeos com álcool (MORIN et al., 2007). A glicerina contém entre 80 a $95 \%$ de glicerol e impurezas, como água, sais, ésteres, álcool, óleo residual, os quais determinam menor valor deste coproduto no mercado (OOI et al., 2004). A glicerina bruta possui $86,95 \%$ de glicerol; $9,22 \%$ de umidade; $0,028 \%$ de metanol; $0,41 \%$ de proteína bruta; $0,12 \%$ de gordura; 3,19\% de matéria mineral; $1,26 \%$ de sódio; $1,86 \%$ de potássio e $3.625 \mathrm{kcal} / \mathrm{kg}$ de energia bruta (LAMMERS et al., 2008). Segundo Südekum (2008), a glicerina pode apresentar teores variáveis de glicerol, água, metanol e ácidos graxos, sendo classificada de acordo com os níveis de glicerol na sua composição (baixa pureza 50 a 70\%, média pureza e alta pureza acima de $99 \%$ de glicerol). Desta forma, o processamento da matéria-prima determina o grau de pureza da glicerina. Neste caso, a glicerina apresentava baixo grau de pureza.

A inclusão de glicerina não influenciou $(\mathrm{P}>0,05)$ a conversão alimentar (Tabela 4). Os resultados obtidos neste trabalho corroboram com Elam et al. (2008) que não observaram diferença na conversão alimentar de bovinos alimentados em confinamento com adição de $0 ; 7,5$ e $15 \%$ de glicerina, respectivamente.

Os coeficientes de digestibilidade da MS, MO, FDN, PB, EE, CNF e CHOT não foram influenciados $(\mathrm{P}>0,05)$ pela inclusão de glicerina (Tabela 5). Os resultados deste trabalho foram superiores aos obtidos por Donkin (2008) que encontrou CDMS $62,7,59,8,61,3$ e $63,1 \%$ e CDFN 34,9; 30,8; 32,4 e $35,2 \%$ para os teores de $0,5,10$ e $15 \%$ de glicerina, respectivamente, em dietas para vacas leiteiras. Entretanto, foram inferiores aos obtidos por Parsons, 
Shelor e Drouillard (2009) para MS (84,9; 84,2 e $84,2 \%)$, MO $(87,1 ; 86,9$ e $86,4 \%)$ e PB $(79,2 ; 79,6$ e $79,1 \%$ ) para as inclusões de 0,2 e $4 \%$ de glicerina na dieta de novilhas terminadas em confinamento.

Por outro lado, a inclusão de glicerina às dietas aumentou de forma linear $(\mathrm{P}<0,05)$ o coeficiente de digestibilidade do extrato etéreo (Tabela 5). As taxas de desaparecimento de glicerina no rúmen podem aumentar com a adaptação dos animais (KREHBIEL, 2008). Essa diferença de digestibilidade do extrato etéreo da dieta pode ser devido à composição da glicerina bruta ou da fonte de matéria prima ou do processo de produção do biodiesel que deu origem a glicerina utilizada. No entanto, deve ser evidenciado que o coeficiente de digestibilidade aparente observado para todos os nutrientes são semelhantes aos observados em animais alimentados a base de concentrado (CALDAS NETO et al., 2000; MARTINS et al., 2000).

Tabela 5. Coeficiente de digestibilidade e suas respectivas equações de regressão e coeficientes de determinação $\left(r^{2}\right)$.

\begin{tabular}{|c|c|c|c|c|c|c|c|}
\hline \multirow{2}{*}{ Parâmetros } & \multicolumn{4}{|c|}{ Níveis de glicerina (\% da MS) } & \multirow{2}{*}{ Equação de Regressão } & \multirow{2}{*}{$\mathrm{r}^{2}$} & \multirow{2}{*}{$\mathrm{CV}(\%$} \\
\hline & G00 & $\mathrm{G} 2,8$ & G6,1 & $\mathrm{G} 9,0$ & & & \\
\hline CDMS & 62,40 & 68,22 & 66,97 & 69,20 & $\hat{\mathrm{Y}}=66,69$ & - & 9,36 \\
\hline CDMO & 66,60 & 68,48 & 68,64 & 69,50 & $\hat{Y}=68,31$ & - & 8,99 \\
\hline CDFDN & 56,70 & 61,57 & 61,01 & 61,68 & $\hat{Y}=60,24$ & - & 7,68 \\
\hline CDPB & 64,77 & 67,91 & 67,41 & 69,05 & $\hat{Y}=67,13$ & - & 9,65 \\
\hline CDEE & 62,45 & 67,07 & 63,38 & 74,48 & $\hat{Y}=61,995+0,975 x$ & 0,59 & 12,90 \\
\hline CDCNF & 77,50 & 75,95 & 76,83 & 78,55 & $\hat{\mathrm{Y}}=77,21$ & - & 7,72 \\
\hline CDCHOT & 67,13 & 68,61 & 69,07 & 69,94 & $\hat{Y}=68,69$ & - & 9,18 \\
\hline
\end{tabular}

Coeficiente de digestibilidade da matéria seca (CDMS), fibra em detergente neutro (CDFDN), proteína bruta (CDPB), extrato etéreo (CDEE) e dos carboidratos não fibrosos (CDCNF).

Fonte: Elaboração dos autores.

\section{Conclusões}

Os resultados deste trabalho mostram que a inclusão da glicerina na dieta de novilhas em crescimento e suplementadas a pasto teve influência negativa no desempenho animal, mas perturbou pouco a digestibilidade das dietas e o bem estar animal. Desta forma, o uso de glicerina em substituição às fontes de energia na dieta de ruminantes depende da qualidade do produto. A glicerina usada apresentava uma coloração mais escura e aspecto oleoso. Estas observações possibilitam caracterizar esta glicerina de baixa pureza.

\section{Referências}

ABDALlA, A. L.; FILHO, J. C. S.; GODOI, A. R.; CARMO, A. A.; EDUARDO, J. L. P. Utilização de subprodutos da indústria de biodiesel na alimentação de ruminantes. Revista Brasileira de Zootecnia, Viçosa, v. 37, p. 260-258, 2008. Suplemento especial.

ABRAHÃO, J. J. S.; PRADO, I. N.; PEROTTO, D.; MOLETTA, J. L. Effects of replacing corn with increasing levels of cassava starch by-products on carcass characteristics and meat for young bulls. Revista Brasileira de Zootecnia, Viçosa, v. 34, n. 5, p. 1640$1650,2005$.

ARICETTI, J. A.; ROTTA, P. P.; PRADO, R. M.; PEROTTO, D.; MOLETTA, J. L.; MATSUSHITA, M.; PRADO, I. N. Carcass characteristics, chemical composition and fatty acid profile of Longissimus musccle of bulls and steers finished in a pasture systems. Asian-Australasian Journal of Animal Science, Seoul, v. 21, n. 10, p. 1441-1448, 2008. 
BERCHIELLI, T. T.; PIRES, A. V.; OLIVEIRA, S. G. Nutrição de ruminantes. Jaboticabal: FUNEP, 2006. 583 p.

CALDAS NETO, S. F.; ZEOULA, L. M.; BRANCO, A. F.; PRADO, I. N.; SANTOS, G. T.; FREGADOLLI, F. L.; KASSIES, M. P.; DALPONTE, A. O. Mandioca e resíduos das farinheiras na alimentação de ruminantes: digestibilidade total e parcial. Revista Brasileira de Zootecnia, Viçosa, v. 29, n. 6, p. 2099-2108, 2000.

CAMPBELL, A. G. Grazed pastures parameters: II. Pasture dry matter production and availability in a stocking rate and grazing management experiment with dairy cows. The Journal of Agricultural Science, Cambridge, v. 67, p. 211-216, 1966.

CHUNG, Y. H.; RICO, D. E.; MARTINEZ, C. M.; CASADY, T. W.; NOIROT, N.; AMES, A.; VARGA, G.A. Effects of feeding dry glycerin to early postpartum Holstein dairy cows on lactational performance and metabolic profiles. Journal of Dairy Science, Champaign, v. 90, n. 8, p. 5682-5691, 2007.

DASARI, M. A.; KIATSIMKUK, P.; SUTTERLIN, W. R.; SUPPES, G. J. Low-pressure hydrogenolysis of glycerol to propylene glycol. Applied Catalysis A: General, New York, v. 281, n. 1-2, p. 225-231, 2005.

D'OLIVEIRA, P. S.; PRADO, I. N.; SANTOS, G. T.; ZEOULA, L. M.; DAMASCENO, J. C.; MARTINS, E. N.; SAKAGUTI, E. S. Effect of replacing soybean meal with canola meal on the performance of Nellore feedlot heifers. Revista Brasileira de Zootecnia, Viçosa, v. 26, n. 3, p. 568-574, 1997.

DONKIN, S. S. Glycerol from biodiesel production: the new corn for dairy cattle. Revista Brasileira de Zootecnia, Viçosa, v. 37, p. 280-286, 2008. Suplemento.

DUCATTI, T.; PRADO, I. N.; ROTTA, P. P.; PRADO, R. M.; PEROTTO, D.; MAGGIONI, D.; VISENTAINER, J. V. Chemical composition and fatty acid profile in crossbred (Bos taurus $x$ Bos indicus) young bulls finished in feedlot. Asian-Australasian Journal Animal Science, Seoul, v. 22, n. 3, p. 433-439, 2009.

ELAM, N. A.; ENG., K. S.; BECHTEL, B.; HARRIS, J. M.; CROCKER, R. Glycerol from Biodiesel Production: Considerations for feedlot diets. Proceedings of the Southwest Nutrition Conference, Tempe AZ, n. 21, 2008.

GARDNER, A. L. Técnicas de pesquisa em pastagem e aplicabilidade de resultados em sistema de produção. Rio de Janeiro: Erca, Gráfica, 1986. 197 p.

KAZAMA, R.; ZEOULA, L. M.; PRADO, I. N.; SILVA, D. C.; DUCATTI, T.; MATSUSHITA, M. Quantitative and qualitative carcass characteristics of heifers fed different energy sources on a cottonseed hulls and soybean hulls based diet. Revista Brasileira de Zootecnia, Viçosa, v. 37, n. 2, p. 350-357, 2008.

KIMURA, F. T.; MILlER, V. L. Chromic oxide measurement, improved determination of chromic oxide in cow feed and feces. Journal of Agricultural and Food Chemistry, Whashington, v. 5, n. 3, p. 216, 1957.

KREHBIEL, C. R. Ruminal and physiological metabolism of glycerin. Journal of Animal Science, Champaign, v. 86, p. 392, 2008. Especial.

LAMMERS, P. J.; KERR, B. J.; WEBER, T. E.; DOZIER, W. A.; KIDDS, M. T.; BREGENDHAL K.; HONEYMAN, M. S. Digestible and metabolizable energy of crude glycerol for growing pigs. Journal of Animal Science, Champaign, v. 86, n. 3, p. 602-608, 2008.

MACH, N.; BACH, A.; DEVANT, D. Effects of crude glycerin supplementation on performance and meat quality of Holstein bulls fed high-concentrate diets. Journal of Animal Science, Champaign, v. 87, n. 2, p. 632-638, 2009.

MAGGIONI, D.; MARQUES, J. A.; PEROTTO, D.; ROTTA, P. P.; DUCATTI, T.; MATSUSHITA, M.; SILVA, R. R.; PRADO, I. N. Bermuda grass hay or sorghum silage with or without yeast addition on performance and carcass characteristics of crossbred young bulls finished in feedlot. Asian Australasian Journal of Animal Science, Seoul, v. 22, n. 2, p. 206-215, 2009.

MARTINS, A. S.; PRADO, I. N.; ZEOULA, L. M.; BRANCO, A. F.; NASCIMENTO, W. G. Digestibilidade aparente de dietas contendo milho ou casca de mandioca como fonte energética e farelo de algodão ou levedura como fonte protéica em novilhas. Revista Brasileira de Zootecnia, Viçosa, v. 29, n. 1, p. 269-277, 2000.

MOREIRA, F. B.; PRADO, I. N.; CECATO, U.; WADA, F. Y.; NASCICMENTO, W. G.; SOUZA, N. E. Suplementação com sal mineral proteinado para bovinos de corte, em crescimento e terminação, mantidos em pastagem de grama estrela roxa (Cynodon plectostachyrus Pilger) no inverno. Revista Brasileira de Zootecnia, Viçosa, v. 32, n. 2, p. 449-455, 2003.

MOREIRA, F. B.; PRADO, I. N.; CECATO, U.; WADA, F. Y.; MIZUBUTI, I. Y. Forage evaluation, chemical composition, and in vitro digestibility of continuously grazed star grass. Animal Feed Science and Technology, v. 113, p. 239-249, 2004.

MORIN, P.; HAMAD, B.; SAPALY, G.; ROCHA, M. G. C.; PRIES DE OLIVEIRA, P. G.; GONZALEZ, W. A.; SALES, E. A.; ESSAYEM, N. Transesterification of rapeseed oil with ethanol. Applied Catalysis A: General, New York, v. 330, p. 69-76, 2007. 
MYERS, W. D.; LUDDEN, P. A.; NAYIGIHUGU, V.; HESS, W. Technical Note: a procedure for the preparation and quantitative analysis of samples for titanium dioxide. Journal of Animal Science, Champaign, v. 82, n. 1, p. 179-183, 2004.

OOI, T. L.; YONG, K. C.; HAZIMAH, A. H.; DZULKEFLY, K.; WAN-YUNUS, W. M. Z. Glycerol residue - a rich source of glycerol and medium chain fatty acids. Journal of Oleo Science, Japan, v. 53, n. 1, p. 29-33, 2004.

PARSONS, G. L.; SHELOR, M. K.; DROUILLARD, J. S. Performance and carcass traits of finishing heifers fed crude glycerin. Journal of Animal Science, Champaign, v. 87, n. 3, p. 653-657, 2009.

PRADO, I. N.; NERY, A. O.; ROTTA, P. P.; PEROTTO, D.; PRADO, R. M.; SILVA, R. R.; SOUZA, N. E.; MOLETTA, J. L. Chemical and fatty acid composition of Longissimus muscle of crossbred bulls finished in feedlot. Asian-Australasian Journal of Animal Science, Seoul, v. 22, n. 3, p. 1054-1059, 2009.

PYATT, A.; DOANE, P. H.; CECAVA, M. J. Effect of crude glycerin in finishing cattle diets. Journal of Animal Science, Champaign, v. 85, p. 412, 2007. Supplement 1. Abstract.

ROTTA, P. P.; PRADO, R. M.; PRADO, I. N.; VALERO, M. V.; VISENATINER, J. V.; SILVA, R. R. The effects of genetic groups, nutrition, finishing systems and gender of Brazilian cattle on carcass characteristics and beef composition and appearance: a review. Asian Australasian Journal of Animal Science, Seoul, v. 22, n. 2, 1718-1734, 2009a.

ROTTA, P.P.; PRADO, I. N.; PRADO, R. M.; MOLETTA, J. L.; SILVA, R. R.; PEROTTO, D. Carcass characteristics and chemical composition of the Longissimus muscle of Nellore, Caracu and Holstein-friesian bulls finished in feedlot. Asian Australasian Journal of Animal Science, Seoul, v. 22, n. 4, p. 598-604, 2009 b.
SCHRÖDER, A.; SÜDEKUM, K. H. Glycerol as a byproduct of biodiesel production in diets for ruminants. Paper No. 241 in New Horizons for Old Corp. Proc. 10 $0^{\text {th }}$ International Rapeseed Congress. Canberra, Australia. N. Wratten and P. A. Salisbury, ed. Regional Institute Ltd., Gosford, New South Wales, Australia. 1997.

SILVA, D. J.; QUEIROZ, A. C. Análise de alimentos: métodos químicos e biológicos. 3. ed. Viçosa: UFV, 2002. $235 \mathrm{p}$.

SNIFFEN, C. J.; O’CONNOR, J. D.; VAN SOEST, P. J.; FOX, D. G.; RUSSEL, J. B. A net carbohydrate and protein system for evaluating cattle diets: IICarbohydrate and protein availability. Journal of Animal Science, Champaign, v. 70, n. 11, p. 3562-3577, 1992.

SÜDEKUM, K. H. Co-products from biodiesel production. In: GARNSWORTHY, P. C.; WISEMAN, J. (Ed.). Recent advances in animal nutrition. Nottingham: Nottingham University Press, 2008. p. 210-219.

TRABUE, S.; SCOGGIN, K.; TJANDRAKUSUMA, S.; RASMUSSEN, M. A.; REILLY, P. J. Ruminal fermentation of propylene glycol and glycerol. Journal Agricultural and Food Chemistry, Davis, v. 55, p. 70437051, 2007.

VAN SOEST, P. J.; ROBERTSON, J. B.; LEWIS, B. A. Methods for dietary fiber, neutral detergent fiber, and non starch polysaccharides in relation to animal nutrition. Journal of Dairy Science, Champaign, v. 74, n. 10, p. 3583-3597, 1991.

VIEIRA, A.; LOBATO, J. F.; CORREA, E. S.; TORRES JUNIOR, A. A.; CEZAR, I. M. Produtividade e eficiência de vacas Nelore em pastagem de Brachiaria decumbens Stapf nos Cerrados do Brasil Central. Revista Brasileira de Zootecnia, Viçosa, v. 34, n. 4, p. 1357-1365, 2005.

WILM, H. G.; COSTElO, O. F.; KLIPPlE, G. E. Estimating forage yield by the double sampling method. Journal of the American Society of Agronomy, Madison, v. 36, p. 194-203, 1944. 
